\title{
Alterstice
}

Revue internationale de la recherche interculturelle

International Journal of Intercultural Research

Revista International de la Investigacion Intercultural

\section{Le choix des mots}

\section{Yvan Leanza}

Volume 5, numéro 1, 2015

URI : https://id.erudit.org/iderudit/1077302ar

DOI : https://doi.org/10.7202/1077302ar

Aller au sommaire du numéro

Éditeur(s)

Alterstice

ISSN

1923-919X (numérique)

Découvrir la revue

Citer ce document

Leanza, Y. (2015). Le choix des mots. Alterstice, 5(1), 1-2.

https://doi.org/10.7202/1077302ar d'utilisation que vous pouvez consulter en ligne.

https://apropos.erudit.org/fr/usagers/politique-dutilisation/ 


\title{
가
}

\section{alterstice}

ÉDITORIAL

\section{Le choix des mots}

\author{
Yvan Leanza $^{1}$
}

La Méditerranée est devenue la frontière la plus mortelle de la planète. Triste record.

Elle engloutit les vies de milliers d'êtres humains fuyant la guerre, les persécutions, la misère, ou plusieurs de ces conditions étroitement entremêlées avec d'autres encore, comme les changements climatiques. Pour qualifier ces personnes, un " nouveau » terme est employé par les médias, mais aussi par les différents professionnels et décideurs qui se prononcent sur ce drame : migrants.

Depuis la nuit des temps, les humains se déplacent pour vivre dans des lieux différents de ceux qui les ont vu naître et grandir. Pour certains penseurs, les migrations sont constitutives de notre humanité. Elles le sont à plus d'un titre. D’abord parce que, physiquement, nous avons dû développer des habiletés pour nous déplacer sur des distances de plus en plus longues pour peupler presque toute la Terre. Ensuite, la rencontre entre peuples différents et la découverte et l'installation dans des environnements aux écologies fort variées ont influencé grandement le développement des innombrables cultures qui ont formé et forment encore l'humanité. Des récits de migration de peuples ou d'individus se trouvent dans de nombreux textes, sacrés ou littéraires, certains vieux de plusieurs millénaires. L'être humain ne serait pas ce qu'il est aujourd'hui sans avoir migré. Nous sommes tous à la fois redevables des migrations de nos prédécesseurs sur cette planète et concernés par les migrations contemporaines, qui touchent un nombre toujours plus important de personnes : plus d'un milliard d'humains seront migrants en 2050 selon le Programme des Nations unies pour le développement (2009). II ne s'agit pas d'un phénomène extérieur qui vient perturber le cours " normal » de la vie des pays du Nord, mais bien d’une réalité incontournable de notre condition humaine.

Migrer signifie changer de lieu de résidence. Tous les $1^{\text {er }}$ juillet, les Québécois migrent « en masse ». C'est en effet la date favorite des habitants du Québec pour déménager. On comprend avec cet exemple que migrer n'implique pas nécessairement de traverser des frontières, de parcourir des milliers de kilomètres ou de changer de langue et d'environnement socioculturel... Les personnes qui effectuent une migration sont des migrants. La forme adjectivale tirée du participe présent employée ici indique un processus en cours, non achevé, ainsi que la simultanéité de cette action (migrer) avec une autre (se reloger, mais cela pourrait aussi être étudier, visiter, travailler, fuir, sauver sa vie, etc.).

Alors pourquoi employer maintenant ce terme pour les personnes qui traversent la Méditerranée (ou d’autres étendues d'eau ailleurs dans le monde) pour sauver leur vie? Peut-être qu'à l'origine de ce changement de vocabulaire, il y avait un souci de rendre compte plus objectivement d'une réalité cruelle. En effet, les " anciens " termes, comme étrangers, sans-papiers, boat-people, demandeurs/requérants d'asile ou réfugiés ont fini par avoir une connotation uniquement négative. Migrant ne fait que qualifier un parcours, sans nécessairement présumer de la qualité de ces personnes et sans indiquer de cause à ce déplacement. Ces personnes n'ont pas non plus de statut officiel en dehors du fait qu'elles se déplacent : pas de visa, pas de permis de séjour, pas d'autorisation d'entrée... De ce fait, l'arsenal du lexique juridique (très variable d'un pays à l'autre) ne peut pas s'appliquer puisqu'elles n'ont pas encore franchi la frontière Schengen. Elles ne sont illégales, demandeuses du statut de réfugié ou de tout autre 
titre de séjour qu'au moment où elles franchissent cette frontière. Avec le terme migrant, le récit de cette tragédie demeure dans une objective neutralité. Est-ce vraiment le cas?

Bien sûr que non. Des qualificatifs accompagnant les comptes rendus et, surtout, les prises de position sur ce drame sont les mêmes que celles que l'on entendait il y a 20 ans sur l'arrivée des demandeurs d'asile fuyant la guerre en ex-Yougoslavie ou les propos haineux à l'égard des non-nationaux, à d'autres périodes de l'histoire de l'Europe. Les politiques européennes actuelles sont orientées sur la répression (des passeurs) et le contrôle des flux migratoires pour les freiner... II y aurait menace d'invasion massive. Les discours populistes sur le trop-plein de réfugiés... pardon... de migrants est remis au goût du jour et les clichés éculés sur l'asile sont à nouveau à l'ordre du jour des débats médiatiques (trop de migrants qui viennent voler nos emplois et profiter des généreuses allocations sociales, qui vont entraîner les finances publiques dans un gouffre, etc.). Le terme a changé, le débat de fond lui est toujours le même. Heureusement, la compassion s'exprime aussi.

Il est un terme qui ne s'utilise plus guère dans le cadre des débats sur les migrations. Peut-être est-il considéré comme désuet. Il est employé presque uniquement pour qualifier certaines personnalités engagées politiquement qui ont dû vivre une migration forcée en raison de cet engagement. Ce terme pourrait retrouver une place de choix. C'est celui d'exilé. L'exil est une mesure pénale ou une contrainte qui implique de devoir quitter le lieu où l'on vit pour une période déterminée ou non. C'est bien ce que vivent toutes ces personnes qui traversent déserts et mers dans l'espoir de vivre dignement. Point n'est besoin de réserver ce terme aux seuls militants, artistes ou intellectuels persécutés. Avec ce terme, on perd peut-être en objectivité, mais on se rapproche un peu de l'expérience (in)humaine de cette migration contemporaine. Le terme nous rappelle aussi que ces mouvements humains ont des causes, pas toujours heureuses. Dans le cas des exilés de la Méditerranée, les causes ne sont pas seulement syriennes, érythréennes ou ouest-africaines, elles sont globales et impliquent aussi les riches pays du Nord.

Ce numéro est consacré aux pères migrants, dont certains ont parfois vécu une migration difficile, à l'image de cet exilé syrien pleurant de joie en débarquant à Kos (Grèce) tout en serrant (protégeant) ses deux enfants, dont la photo, prise par Daniel Etter et publiée dans le New York Times, circule dans les médias sociaux.

"Tout le monde sait comment on fait les bébés

Mais personne sait comment on fait des papas » - Stromae

Souhaitons que ces quelques textes puissent faire démentir, au moins partiellement, les paroles du chanteur belge (mais pas seulement).

Bonne lecture!

\section{Références}

Stromae. (2013). Papaoutai [enregistrée par Stromae]. Sur Racine Carrée. Paris : Mercury, Universal Music.

Programme des Nations Unies pour le Développement. (2009). Human Development Report 2009. Overcoming barriers: Human mobility and development. New York : Palgrave Macmillan.

\section{Rattachement de l'auteur}

${ }^{1}$ Université Laval, Québec, Canada

\section{Correspondance}

alterstice@gmail.com

\section{Pour citer cet article}

Leanza, Y. (2015). Le choix des mots [Éditorial]. Alterstice, 5(1), 1-2. 